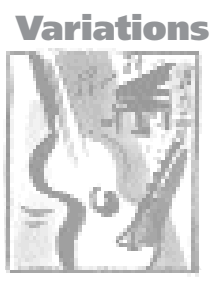

\title{
La compétitivité et l'égalitarisme allemands et japonais à l'épreuve
}

\author{
par Gregory Jackson
}

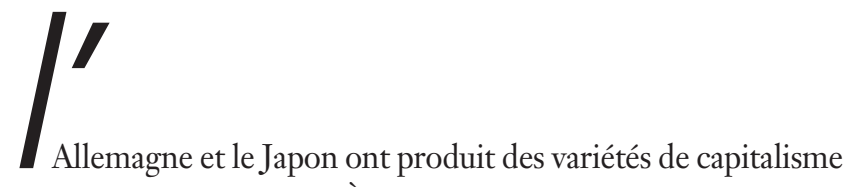

alliant compétitivité et une certaine égalité. À la différence des pays anglo-saxons, le marché y est étroitement inscrit dans le social. Cette configuration, qui présente, en termes d'incitation et d'allocation des ressources, une efficacité moindre que celle offerte par le marché, offre en revanche des sources non économiques de confiance ou d'obligations réciproques qui favorisent le développement. Ces « contraintes fécondes » (Streeck 1997) contribuent à limiter l'opportunisme et les coûts de transaction élevés qui caractérisent les capitalismes de marché « purs », tout en garantissant coopération, X-efficacité et capacités collectives et organisationnelles à long terme. Les deux pays ont choisi la voie dite high road (produits de qualité, travailleurs qualifiés et bien payés), et se sont ainsi imposés sur certains créneaux du marché international. L’Allemagne s'est spécialisée dans la «production de qualité diversifiée », le Japon dans la « production de masse flexible ». En outre, 
la limitation des forces du marché a contribué à contenir l'inégalité des revenus ${ }^{1}$.

Allemagne et Japon forment le plus souvent, dans les travaux portant sur les variétés de capitalisme, un couple opposé à celui de la Grande-Bretagne et des ÉtatsUnis : États contre marchés, économies coordonnées contre économies non coordonnées, systèmes organisés contre systèmes sans organisation, capitalisme inscrit ou non dans le social, développement tardif ou précoce, modèle rhénan contre modèle anglo-américain (Boyer 2000). Pourtant, s'il existe entre eux des analogies fonctionnelles incontestables, ces deux pays diffèrent structurellement dans leur manière de brider la puissance du marché.

Le modèle « développementaliste » du Japon cherchait à rattraper l'Occident par une coopération entre État, employeurs et salariés, qui empruntait au moins trois voies (Streeck et Yamamura 1996). D'abord, des relations étroites unissent le Parti libéral-démocrate dominant, l'administration (surtout le ministère du Commerce extérieur et de l'Industrie, et celui des Finances) et les milieux industriels. Cette coopération vise à diffuser la technologie, à protéger les secteurs faibles et à guider l'allocation du crédit. La politique industrielle « guide » aussi l'ajustement sectoriel en opérant une certaine redistribution, en orientant la concurrence et en contribuant à la fourniture de biens collectifs. Ensuite, les keiretsu (groupes d'entreprises) ont formé des réseaux de coopération. Les keiretsu horizontaux, comme le groupe Mitsubishi, sont constitués de firmes de différentes branches, liées par des participations croisées coordonnées par une banque principale. La participation croisée protège les dirigeants de la pression des actionnaires « extérieurs » et abaisse les coûts de transaction et les risques en facilitant certaines joint ventures (R\&D, distribution à l'étranger, etc.). Les keiretsu verticaux forment des réseaux entre sociétés mères et firmes sous-traitantes (propriété commune, transfert de technologie, échange de personnel) permettant la production à flux tendu. Troisièmement, les relations de travail dans les grandes sociétés sont caractérisées par l'emploi à vie, la rémunération à l'ancienneté - qui poussent l'entreprise à investir dans des qualifications très spécifiques - et la participation aux décisions de syndicats « maison ». Tout en limitant la souplesse de l'emploi en termes d'effectifs, ce système favorise la mobilité fonctionnelle des salariés ainsi que la qualité de la production et l'innovation graduelle au niveau de l'atelier.

L'« économie sociale de marché » instituée par l'Allemagne après la guerre, elle, a construit un compromis historique complexe entre plusieurs courants du libéralisme, de la social-démocratie et du catholicisme (Lehmbruch 2000). Les autorités y favorisent la coopération des associations privées, en particulier des organisations patronales et syndicales. Ainsi les négociations collectives sont conduites au niveau de l'ensemble d'une branche par ces organisations. D'où des salaires à la fois élevés et présentant très peu d'écart d'une firme ou d'une branche à l'autre, ce qui a protégé les entreprises de la concurrence salariale et les a encouragées à 
élever les niveaux de qualification. Les partenaires sociaux organisent aussi une formation professionnelle caractérisée par des profils aisément transférables d'une firme à l'autre. Au niveau des firmes, la coopération est institutionnalisée dans la corporate governance : le capital et le travail sont représentés paritairement aux conseils de surveillance, et les conseils d'usine sont mandatés pour représenter les intérêts des salariés au sein de la firme. À cette « citoyenneté industrielle » des travailleurs s'ajoute l'« actionnariat patient », constitué par exemple d'entreprises industrielles et de banques, qui protège les directions de la pression des actionnaires de court terme. Enfin, l'État-providence offre un niveau élevé de « citoyenneté sociale » et une limitation des inégalités qui ont souvent facilité la négociation de réductions d'emplois entre les partenaires sociaux. En somme, on peut schématiser ainsi les différences entre les deux pays : institutions « de solidarité » en Allemagne, enracinées dans un corporatisme spécifique qui attache l'acteur privé à son association, institutions « segmentaires » au Japon, où la coopération se nourrit d'engagements mutuels à l'intérieur des réseaux d'entreprise (Thelen et Kume 2000b).

Durant les années quatre-vingt, les excellentes performances de l'Allemagne et du Japon ont suscité beaucoup d'intérêt, mais tout a changé par la suite. Les points naguère considérés comme forts apparaissent aujourd'hui souvent comme des faiblesses. En effet, les deux pays ont connu, entre 1985-1990 et 1991-1997, une chute brutale de leurs excédents commerciaux (de 5 à 0,7 \% du PIB en Allemagne, de 2,5 à 1,6 \% au Japon) et de leur part des exportations mondiales. Le coût de l'heure de travail est devenu moins compétitif², et la croissance du PIB par habitant est tombée en dessous de celle des États-Unis. Le Japon a souffert de l'éclatement de la bulle financière et de la crise bancaire, tandis que le chômage augmentait rapidement en Allemagne. On attribue souvent cette crise au renouveau de l'efficacité des marchés internationaux en termes d'allocation des ressources. L'X-efficacité et l'innovation graduelle sont-elles de bien puissants atouts dans des activités telles que les biotechnologies ou les technologies de l'information?

L'Allemagne et le Japon convergeront-ils vers un modèle de marché libéral ? La question reste ouverte. La recherche comparative permet au moins de faire un constat important : même si les régimes se rapprochent, la spécificité institutionnelle des différents modèles nationaux produit des problèmes et des trajectoires de changement également spécifiques.

\footnotetext{
1. Au milieu des années quatre-vingt-dix, l'Allemagne et le Japon avaient des coefficients de Gini (mesurant l'inégalité globale de revenu) de 28,2 et 26,5 respectivement, contre 34,6 en Grande-Bretagne et 34,4 aux États-Unis. Les indices de la dispersion des revenus montrent que l'Allemagne est le seul pays de l'OCDE dont l'inégalité diminue depuis les années quatre-vingt (Jackson 2000).

2. Sur la même période et en dollars, le salaire horaire en Allemagne est passé de $117 \%$ à $136 \%$ du salaire américain, et celui du Japon de 77 \% à 115 \% (US Department of Labor, Bureau of Labor Statistics, Supplementary Tables, News Release USDL 98-376, septembre 1998).
} 


\section{Cercles vertueux, cercles vicieux}

Les « modèles nationaux » de capitalisme doivent s'analyser en termes de relations et de complémentarités entre les institutions existantes au sein d'une économie nationale : l'efficacité d'une institution est rarement absolue. En réalité, « telle institution réussira dans un domaine donné parce que telle autre institution sera présente dans un autre domaine $»(\text { Aoki } 2000: 8)^{3}$. Ces interdépendances ont produit des « cercles vertueux » tout au long des années quatre-vingt. Mais elles peuvent aussi créer des « cercles vicieux » lorsque les conditions extérieures changent ou que l'on atteint un point de rendement décroissant (Jürgens 2000). Politiquement, les modèles s'apparentent moins à un système. Les communautés créées par telle politique nationale se « sectorisent » par la segmentation de l'État et des acteurs sociaux (Lehmbruch 2000). Les États et les organisations diffèrent par leur capacité à coordonner les politiques publiques entre différents secteurs. Un changement marginal dans un domaine peut avoir des conséquences déstabilisantes dans un autre. Inversement, des efforts de réforme peuvent être bloqués par des intérêts établis ailleurs. Les médiocres capacités de coordination sectorielle en Allemagne et au Japon sont un facteur de leur relative stabilité dans les années quatre-vingt-dix, mais peut-être aussi de leur stagnation ultérieure.

Quelques-uns de ces changements sectoriels peuvent être développés à titre d'exemples :

1. Systèmes financiers. Les systèmes financiers de ces deux pays sont fondés sur la banque. Le secteur bancaire est puissant et détient en dépôt une large part de l'épargne nationale ; la capitalisation boursière est faible, ainsi que le nombre de sociétés cotées ; la part d'actifs sécurisés dans le secteur entrepreneurial est étroite; enfin, les fonds de pension privés sont marginaux (Jackson et Vitols 2000) ${ }^{4}$. Ce contraste avec les systèmes de financement fondés sur les marchés se retrouve dans les stratégies du risque et de la rentabilité, ainsi que dans le poids de l'État dans l'allocation du crédit.

Au cours de la dernière décennie, le rapprochement des écarts de taux d'intérêt a érodé les profits bancaires, et les réformes politiques du secteur financier ont ouvert la concurrence sur de nouveaux segments de marché. L'Allemagne a ainsi adopté plusieurs « lois de promotion des marchés financiers » établissant une agence de régulation (Bundesaufsichtsamt für Wertpapierhandel), copiée sur la Commission des opérations de bourse des États-Unis, pour surveiller les échanges entre insiders et libéraliser les fonds mutuels (Lütz 1999). Le Japon, lui, a connu de graves problèmes bancaires après l'éclatement de la bulle. Le « big bang » japonais a fait tomber les barrières entre les activités financières, modifié le système de brevets et créé une agence de régulation bancaire indépendante. La déréglementation a mis fin au pilotage administratif de l'allocation des crédits au profit d'un 
système davantage fondé sur des règles. Mais aucun des deux pays n'a abandonné ses traditions nationales en la matière ni ne s'est totalement converti à un régime de réglementation libéral.

On trouve derrière ces réformes différentes coalitions (Vitols 2000). Les grandes banques et les sociétés internationales seront les bénéficiaires les plus probables de la libéralisation, quoique la crise bancaire ait rendu prudentes les banques japonaises. La Deutsche Bank, par exemple, évolue rapidement vers des services financiers sophistiqués (prestation de services orientées sur le marché) caractéristiques des banques d'investissement américaines, par le biais d'acquisitions étrangères et de restructuration interne. Les critiques qu'elle s'est attirées à propos de son rôle dans la fusion Thyssen-Krupp ${ }^{5}$ montrent que les relations existant entre les « bouse banks » et les firmes éveillent bien vite le soupçon de conflit d'intérêts, et que la transition est difficile d'une activité bancaire orientée par les relations à une activité orientée par le marché. Inversement, vont souffrir de la réforme : les banques plus petites ou détenues par l'État, les sociétés d'assurance, certains secteurs industriels et certains départements ministériels. Au Japon, le calendrier de la déréglementation et de la libéralisation a joué un rôle dans la crise bancaire : l'industrie ayant réduit ses emprunts, les banques se sont mises à prêter à des entreprises plus petites et plus risquées, qui garantissaient leurs emprunts par des actifs surévalués (Hoshi et Kashyap 1999).

En somme, les systèmes financiers fondés sur la banque sont en voie de transformation tant en termes de réglementation que des stratégies déployées par les acteurs vis-à-vis du risque et de sa rémunération. Le plus important est peut-être l'exigence de transparence (garantie par les normes internationales de comptabilité) qui réduit l'avantage informationnel des insiders. On va vers un système à la fois plus orienté vers le marché et plus différencié, le secteur public gardant la structure ancienne où domine la banque tandis que les grandes sociétés privées se financeront de plus en plus sur fonds propres.

2. Les systèmes de retraite. Le système de retraite a une forte influence sur la demande de produits financiers (Jackson et Vitols 2000 ; Vitols 2000). Aux ÉtatsUnis et au Royaume-Uni, les fonds de pension privés par capitalisation sont les plus

\footnotetext{
3. Ainsi les atouts du Japon s'appuient sur des complémentarités entre le système financier fondé sur la banque, une gestion orientée par l'actionnariat d'entreprise, l'emploi à vie, le syndicalisme « maison » et le pilotage administratif par des ministères. L'Allemagne présente des complémentarités entre le régime de formation professionnelle, la négociation collective, la cogestion et le système financier fondé sur la banque.

4. La capitalisation boursière représentait $31 \%$ du PIB en Allemagne, $62 \%$ au Japon et $101 \%$ aux États-Unis en 1997. Les dépôts bancaires des ménages étaient deux à trois fois plus élevés dans ces deux pays qu'aux États-Unis, de même que le ratio actifs du secteur bancaire sur PIB.

5. L'acquisition était prônée par deux de ses filiales (Morgan Grenfell et Kleinwort Benson) alors qu'elle-même siégeait aux conseils de surveillance de Krupp et de Thyssen.
} 
grands acheteurs nets d'actions, et leurs actifs représentent respectivement $66 \%$ et $73 \%$ du PIB. Les systèmes de pension par répartition, fondés sur la solidarité entre générations à l'échelle de l'ensemble de la société, de l'entreprise ou de la famille, suscitent une faible demande de titres. Or les systèmes publics de ce type représentent une large part des pensions servies en Allemagne et au Japon (environ $80 \%$, contre $68 \%$ aux États-Unis). Les systèmes privés restent de taille modeste et se coulent dans l'organisation générale, à l'instar du livret de retraite d'entreprise en Allemagne ou des portefeuilles très stables des sociétés japonaises d'assurance sur la vie. Les fonds de pension avec responsabilités fiduciaires n'atteignent pas $10 \%$ du PIB dans ces deux pays.

Mais les systèmes publics sont confrontés au problème du vieillissement de la population. En Allemagne, les cotisations de retraite et d'autres assurances sociales sont passées de 26,5\% du salaire brut en 1970 à plus de $40 \%$ dans les années quatrevingt-dix (Manow 2000). L'augmentation des charges sociales qui en résulte est un obstacle majeur à la croissance des emplois et pousse les entreprises à rationaliser leur poste main-d'œuvre. Au surplus, les employeurs et les syndicats se sont, de fait, entendus pour faire peser le coût des restructurations sur le système public de pension, par la pratique de la retraite anticipée (Hassel 2000). Deux problèmes supplémentaires s'y ajoutent : d'abord, le modèle social conservateur de l'Étatprovidence allemand tend de fait, par de multiples biais, à réduire la population au travail et la natalité ; ensuite, le secteur financier s'intéresse fortement au potentiel du marché des fonds de pension privés. Il y est d'ailleurs encouragé par l'Union européenne, avec sa directive sur la libéralisation du capital qui favorisera la compétition internationale dans l'assurance sur la vie et les systèmes privés de retraite. L'érosion du système public de retraite aura des conséquences sur d'autres aspects du modèle allemand : plus forte demande de produits financiers, évolution de la structure de la propriété, pression accrue des actionnaires de court terme sur la direction des entreprises, moindre acceptation des réductions d'emploi.

Au Japon, le système public de retraite n'est pas soumis à une telle pression de coût et n'est pas utilisé aussi massivement pour l'ajustement de l'emploi : au lieu de les mettre en retraite anticipée, on transfère les travailleurs en surnombre dans de petites entreprises du même keiretsu. Quant au système privé, il est confronté à la baisse des retours sur actifs après la bulle. Les sociétés d'assurance sont organisées en sociétés mutuelles. Actionnaires stables des groupes industriels, elles ne cherchent pas à jouer sur la valeur des actions et se contentent de dividendes réguliers. Mais ces arrangements risquent d'être remis en cause : une masse croissante de plans de pension arrive à maturité alors même que les dividendes sont passés au-dessous des taux d'intérêt et que les bénéfices tirés des transactions sur le marché sont négatifs depuis plusieurs années. Les assureurs sont donc amenés à restructurer leurs portefeuilles et à envisager une attitude plus active. 
3. Corporate governance. Les modèles allemand et japonais, fondés sur la banque et l'actionnariat d'entreprise, favorisent d'une part l'engagement financier à long terme du capital par des réseaux d'entreprises et de banques tissés d'une multiplicité de relations et d'incitations mutuelles, d'autre part la « citoyenneté industrielle » des salariés assurée par la cogestion ou l'emploi à vie (Jackson 2000). Ces complémentarités institutionnelles produisent un socle de confiance et d'engagement chez toutes les parties prenantes, sur lequel se bâtit une capacité organisationnelle de longue haleine autorisant une production de qualité par des travailleurs hautement qualifiés. Une des principales conditions en est l'absence de marchés pour le contrôle des entreprises (De Jong 1996).

Or plusieurs indices laissent penser que l'engagement financier s'effrite (Jackson 2000). Les liens entre banque et industrie se distendent. Les grandes entreprises s'appuient de moins en moins sur le crédit bancaire ${ }^{6}$, et les banques ont commencé à restructurer leur portefeuille, abandonnant progressivement l'actionnariat stable au profit d'une stratégie plus sélective et plus soucieuse de performance. Deuxièmement, les groupes industriels se relâchent lentement. Au Japon, la part d'actionnariat stable des sociétés cotées est passée de 41,5\% en 1987 à 35,7 \% en 19977. En Allemagne, la propriété interentreprise et bancaire est restée relativement stable, mais vingt-quatre des cent plus grandes firmes ont une structure de propriété fragmentée ${ }^{8}$. Il est probable que cette déconcentration va se poursuivre avec la multiplication des fusions et les avantages supplémentaires que les entreprises vont en tirer. Troisièmement, les investisseurs institutionnels, tant étrangers que nationaux, qui recherchent avant tout la performance immédiate sont de plus en plus présents. Durant les années quatre-vingt-dix, la propriété étrangère est restée de l'ordre de 11-12 \% en Allemagne, mais est passée de $4 \%$ à $10 \%$ au Japon. Les investisseurs étrangers liquides, qui s'intéressent bien plus à la performance du marché qu'aux relations organisationnelles de longue haleine, prennent largement part au turn-over des actions. Quatrièmement, les fusions et acquisitions transfrontalières se multiplient. Stimulées par le marché européen, les entreprises allemandes sont très actives (Daimler Benz, Hoechst, Deutsche Bank, Commerzbank, Mannesmann...). Les sociétés japonaises, à la recherche d'un meilleur accès aux marchés internationaux, sont entrées dans des alliances stratégiques avec des

\footnotetext{
6. Au Japon, une enquête du ministère des Finances montre que le taux de financement interne est passé de $65 \%$ à $94 \%$ entre 1992 et 1996, avec une baisse correspondante des emprunts bancaires de 33 \% à environ $1 \%$ des fonds nouveaux. 7. La participation croisée est passée de 21,2 \% à 18,2 \% sur la même période, bien que les six principaux keiretsu présentent des évolutions différentes. Mitsubishi a conservé sa structure, tandis que l'actionnariat stable de Fuji est passé de 54,6 \% à $38,2 \%$ et les participations croisées au sein du groupe de $39,9 \%$ à $23,4 \%$.

8. Les banques disposent néanmoins toujours d'une large part des voix grâce au système de procuration, et détiennent des sièges aux conseils de surveillance.
} 
sociétés étrangères. Or ces alliances sont susceptibles d'éroder la cohésion interne des keiretsu et de déposséder l'État d'une partie de ses moyens d'action sur des firmes qui se déploient désormais dans le monde entier.

Pourtant, la réforme institutionnelle de la corporate governance est modeste. Tout d'abord, les plus grands changements se produisent dans les domaines dépolitisés de la comptabilité et de la transparence, où la mise aux normes internationales (c'està-dire américaines) est en cours. La fixation de la valeur par le marché et la transparence n'ont pas d'impact immédiat sur la corporate governance, mais donnent aux actionnaires des atouts pour faire valoir leurs intérêts. Par exemple, les firmes allemandes peuvent toujours conserver les normes comptables nationales, mais doivent adopter les normes américaines pour pouvoir être cotées à la bourse de New York. Deuxièmement, l'Allemagne et le Japon ont libéralisé l'usage des stock options, ce qui incite les dirigeants à agir dans le sens des intérêts des actionnaires et élargit les différences de revenu entre cadres de direction et employés. Troisièmement, si les associations patronales japonaises sont favorables à des réformes modestes améliorant la transparence et l'indépendance des auditeurs, elles ne le sont guère à des mesures plus hardies telles que le recrutement externe d'une partie des dirigeants. Les sociétés de holding pur sont désormais autorisées, pour la première fois depuis 1945, et pousseront certainement à des restructurations selon des critères plus financiers9. En Allemagne, la réforme des conseils d'administration visait surtout à réduire l'influence des banques. La version SPD de la KontraG (loi sur la transparence et le contrôle des sociétés) a été édulcorée par les gouvernements CDU. Les syndicats se sont aussi opposés à la réforme proposée des conseils d'administration à cause de son impact potentiel sur la cogestion ${ }^{10}$.

L'alignement de plus en plus marqué sur l'intérêt des actionnaires a un effet sur le rôle des salariés dans la corporate governance. Les grandes sociétés dégraissent « en douceur » par des mesures d'ajustement de l'emploi. Au Japon, de plus en plus de firmes connaissent de tels ajustements à cause de la récession, de l'appréciation du yen, de l'expansion trop rapide de l'époque de la bulle, et de la réduction des sociétés mères. Mais la plupart d'entre elles ont évité les licenciements et négocié des ajustements de l'emploi : limitation des heures supplémentaires (29,6\%), arrêt du recrutement $(27,4 \%)$, mutation interne $(23,1 \%)$ ou vers des filiales $(20,2 \%)$, départ en retraite (3,7 \%) ou démission (1,9\%) volontaires (JIL 1995). En Allemagne, les conseils d'usine ont fait des concessions en échange de la préservation

\footnotetext{
9. Peu de sociétés sont prêtes à adopter les structures des holdings tant que le paiement consolidé des impôts n'est pas autorisé et que le code de commerce ne permet pas les échanges d'actions.

10. La KontraG élargissait modestement les devoirs d'information de la direction, augmentait le nombre annuel de réunions obligatoires du conseil de surveillance de deux à quatre, et comptait double le siège de président dans la détermination du nombre maximum de sièges au conseil.
} 
des emplois ou de l'investissement sur place : baisse des normes sociales, élimination des dépassements par rapport aux salaires collectivement négociés, réduction des sursalaires pour heures supplémentaires ou travail par équipes, etc. (Kotthoff 1998). La citoyenneté industrielle et la cogestion se heurtent néanmoins à l'amoindrissement de la capacité de la loi à modeler l'autorité managériale, qui découle de la réorganisation rapide des sociétés et de la pression croissante des actionnaires. L'emploi à vie japonais est, lui aussi, de plus en plus problématique à mesure que le ralentissement de la croissance et la baisse de la rentabilité rendent plus difficiles les compromis entre parties prenantes. À cet égard, la cogestion à l'allemande souffrira sans doute moins de l'alignement sur l'actionnaire, du fait que l'expression des salariés y a une base légale, contrairement au cas japonais où elle est informelle et découle surtout de la faible influence des actionnaires.

Ces nouveaux éléments sont-ils absorbés dans les pratiques existantes ou créentils des pressions supplémentaires sur le reste du modèle ? Dans les deux cas, il s'agit en fait d'une tentative d'hybridation du modèle traditionnel de la coopération employeurs-salariés avec les impératifs d'alignement sur les intérêts de l'actionnaire. Cette hybridation entraîne une différenciation accrue des modalités de la corporate governance. Le marché boursier n'est pas le seul facteur de changement. La direction peut être poussée à aller au-devant des intérêts des actionnaires par la concurrence que rencontrent ses produits plus que par le marché des capitaux. Si les grandes corporations utilisent rarement le financement direct par capitaux propres, le prix des actions peut jouer un rôle croissant dans la stratégie de fusion/acquisition par le biais de fusions sans transfert monétaire, financées par échange de parts. La valeur de l'action sur le marché peut aussi permettre d'arracher des concessions aux représentants ouvriers. De même, l'orientation sur l'actionnaire peut faire évoluer les formes de coopération entre firmes et donner trop d'importance au profit à court terme. L'évolution future dépendra sans doute de l'établissement ou non de marchés ouverts permettant le contrôle des sociétés. Si les deux systèmes sont restés épargnés par les acquisitions hostiles, la réussite de l'achat de Mannesmann par Vodaphone représente un tournant décisif.

4. Relations employeurs-salariés et marché de l'emploi. On considère souvent que l'internationalisation menace particulièrement les relations du travail, dont l'équilibre est bouleversé par la mobilité du capital : ce dernier étant toujours prêt à fuir, les employeurs disposent d'un argument de poids dans la négociation des salaires et des conditions de travail. Inversement toutefois, la compétition internationale peut aussi accroître la valeur des qualifications et rendre les employeurs plus demandeurs de relations du travail consensuelles. Thelen et Kume (2000b) soutiennent ainsi que « l'on trouve une fraction importante de travailleurs (Japon) et de firmes (Allemagne) pour lesquels les arrangements traditionnels en matière de relations du travail s'appliquent et où les employeurs cherchent même à intensifier 
leur coopération avec les salariés au sein de la structure existante. [...] Toutefois, dans les deux pays, cette intensification de la coopération s'accompagne de l'exclusion d'un nombre croissant de firmes et de travailleurs des protections traditionnelles ».

À cet égard, la situation n'est pas la même dans les deux pays. En Allemagne, la négociation collective sert à réduire les différences de salaires d'une firme à l'autre, à supprimer la concurrence sur les salaires et à exercer sur les firmes une pression pour accroître leur productivité. Plusieurs facteurs expliquent la diminution de ce champ d'action : baisse de la solidarité entre employeurs petits et gros, inefficacité des lock-out dans les conflits du travail, intégration très inégale des entreprises est-allemandes. Mais, à mesure que la négociation collective se rétrécit à un noyau de grosses entreprises capables de payer des salaires élevés, les petites seront incitées à quitter les associations patronales. En outre, comme il a été dit plus haut, l'externalisation vers l'État-providence du coût de l'ajustement de l'emploi alourdit les charges salariales. Ces coûts ne peuvent être supportés par les secteurs de bas salaires et de faible productivité, et le chômage va s'accroître (Manow et Seils 2000). Au Japon, nombre d'employeurs et de syndicats s'efforcent ensemble de maintenir l'emploi durable en aménageant le système de salaire à l'ancienneté pour créer un marché de la main-d'œuvre plus compétitif pour les jeunes tout en réduisant le coût du maintien en poste des plus âgés. Un changement du système des salaires mettrait à rude épreuve la solidarité des salariés à l'intérieur des syndicats d'entreprise et risquerait de nuire aux incitations liées au système de formation «maison ». Ces nouveaux clivages n'opposent donc pas la direction aux travailleurs mais certaines catégories de firmes à d'autres, ou des travailleurs d'âges et de niveaux de qualification différents. Quel sera leur effet à long terme sur le cœur des relations entre directions et travailleurs?

5. Innovation. L'Allemagne et le Japon ont en commun un penchant pour l'innovation à la marge plutôt que pour la quête de grandes percées technologiques fondées sur la recherche. Cette innovation à petits pas se fonde sur le haut niveau de qualification dans les ateliers, lui-même issu, au Japon, du marché intérieur du travail et de la formation sur le tas et, en Allemagne, des qualifications plus larges produites par le système d'apprentissage (Thelen et Kume 2000a). Les deux pays se sont spécialisés, à l'échelle mondiale, dans des produits incorporant une forte composante des arts du technicien et de l'ingénieur. Les études de parts de marché par branche et les enregistrements de brevets confirment que ces modes sont très différents de ceux des États-Unis, qui misent sur les biotechnologies et les technologies de l'information.

Si ces derniers ont connu une stagnation de leur production industrielle dans les années quatre-vingt, la « révolution numérique » leur a redonné l'avantage. Non seulement par suite de leur puissance scientifique, mais aussi de certains traits institutionnels : marché du travail extérieur flexible, marché des capitaux disposé 
au risque, faible pression fiscale. Malgré quelques succès, ces secteurs font pâle figure, comparativement, au Japon et en Allemagne.

Les deux pays ne se heurtent pas aux mêmes difficultés. Les méthodes japonaises de « production de masse flexible »s'appuient sur des gains de productivité fondés sur l'échelle, tandis que la « production diversifiée de qualité » allemande reste une exception en ce qu'elle n'a que peu d'effet d'échelle observable (Boyer 2000). Les exportations japonaises sont concentrées sur des secteurs peu nombreux : automobile, électroménager, équipement électrique. Le Japon a associé forte innovation sur les procédés et innovation marginale sur les produits pour fournir de la qualité supérieure à prix plus bas : le ralentissement de la croissance a donc porté un coup à l'augmentation de la productivité, et la fixation des prix en fonction du marché a érodé les marges. Les exportations allemandes concernent des secteurs plus divers mais cherchent à éviter la compétition par les prix en migrant vers des niches de plus haute qualité, qui y sont moins sensibles : là, l'érosion d'une compétitivité qui n'était pas fondée sur le prix n'a guère eu d'effet sur la productivité.

Certes, les économies libérales ont eu de bonnes performances lors de la percée des nouvelles technologies de l'information; mais les économies non libérales ne sont-elles pas en train de profiter de la phase de maturation de ces technologies pour retrouver des forces (Yamamura 2000) ? L'innovation marginale y revient à l'ordre du jour dans un large éventail de branches, avec la création de produits « hybrides » combinant technologies anciennes et nouvelles. L'atout de la coopération et des ressources collectives pourrait retrouver là tout son poids, les qualifications intermédiaires et la spécialisation technique garder toute leur importance.

\section{Convergence ou hybridation ? Un nouveau pacte d'efficacité et de qualité}

Le capitalisme non libéral est aux prises à la fois avec la pression internationale et avec des problèmes engendrés par les modèles nationaux eux-mêmes. Les points forts de l'Allemagne et du Japon ne font plus recette. Pour autant, on n'assiste pas à une convergence des deux « modèles » vers celui des États-Unis, mais à une hybridation : des éléments de libéralisme sont sélectivement introduits dans des contextes non libéraux. La libéralisation, ce sont notamment des règles uniformisant le champ économique par la transparence de l'information et les relations impersonnelles. Elle œuvre donc contre la coopération entre insiders et contre les compromis de répartition que celle-ci implique. On se réjouira peut-être de voir sombrer les structures corporatistes de Japan Inc., reste que ces institutions ont aussi limité l'inégalité des revenus et les coûts sociaux qui lui sont liés tels que la criminalité, les problèmes de santé publique, l'inégalité des chances.

Le capitalisme non libéral n'est pas simplement placé devant le choix « égalité ou efficacité », car son rapprochement trop rapide du modèle libéral non seulement 
nuirait à l'égalité mais pourrait saper les bases de sa compétitivité à long terme. S'orienter davantage sur le marché (priorité aux intérêts des actionnaires, mobilité de la main-d'œuvre, etc.) peut être rationnel pour des acteurs individuels mais ne garantit pas une performance globale meilleure. Il n'est pas sûr que de nouvelles institutions retrouvent la « complémentarité » des anciennes. L'ajustement aux institutions libérales va probablement créer des frictions et modifier les fonctions d'autres institutions qui leur sont liées. Par exemple, en donnant la priorité à l'actionnaire, les firmes peuvent devenir plus compétitives sur le marché des capitaux mais affaiblir, par un enchaînement de conséquences, les dispositifs de la formation professionnelle. Ou la libéralisation du marché de la main-d'œuvre non qualifiée peut nuire à l'emploi dans des secteurs cruciaux. Je reviens ici sur deux des antagonismes examinés dans cet article, dont les difficultés appelleront des réponses du capitalisme non libéral.

1. Secteur international contre secteur intérieur. La libéralisation conduit à une hétérogénéité croissante entre secteurs et entre entreprises. Les firmes tournées vers l'international adopteront des règles et des pratiques de plus en plus éloignées de celles des firmes plus petites et orientées sur le marché intérieur. Les instruments de politique publique visant à protéger de tels secteurs subissent des pressions croissantes (de l'Union européenne contre les subventions allemandes, des États-Unis contre la protection exercée par le MITI sur l'industrie japonaise). En outre, les efforts pour aider les secteurs internationalisés à rester compétitifs peuvent avoir des effets non désirés sur d'autres secteurs. Par exemple, les efforts pour stimuler le capital risque en vue de l'innovation peuvent placer d'autres secteurs sous une pression indésirable des marchés de capitaux. Ou les marchés financiers libéraux peuvent exercer des pressions d'ajustement sur les banques publiques et leurs clients.

2. Travailleurs du noyau dur et travailleurs de la périphérie. Les institutions du marché et des relations du travail n'ont guère changé. Mais l'emploi dans le secteur central se rétrécit. L'Allemagne est prise dans une spirale de chômage : coût salariaux élevés, rationalisation « négociée » de la production industrielle de qualité, croissance très lente de l'emploi dans les services. Le chômage a atteint 9,8 \% en 1998, contre 4,5 \% aux États-Unis et 6,9 \% en moyenne dans les pays de l'OCDE. Si la « rationalisation négociée » aide les plus grandes firmes à rester compétitives sur les marchés mondiaux, le secteur de la production de qualité par des salariés bien payés (bigh road), en régression, ne crée plus assez d'emplois et d'externalités positives pour soutenir l'économie nationale. L'emploi ne devrait croître que dans le secteur des services de faible productivité, qui rencontre des problèmes de coûts salariaux du fait des charges salariales et de l'alignement des augmentations de salaires sur celles des secteurs productifs. Au Japon, ce problème est moins grave parce que les relations du travail et l'État-providence n'y sont pas aussi 
étroitement liés (autrement dit, l'économie japonaise est déjà plus « duale » que l'allemande). Le chômage reste très bas, passant de 2,1 \% en 1990 à 4,1 \% en 1998. Mais la restructuration des firmes pour améliorer le profit peut conduire au licenciement de la main-d'œuvre de réserve des grosses entreprises. L'affaiblissement des réseaux interentreprises rend plus problématique le transfert négocié de travailleurs dans des firmes et des branches moins productives. Étant donné l'étroite relation qui existe entre l'emploi à vie, les qualifications maison et le syndicalisme d'entreprise, les atouts de la gestion à la japonaise peuvent être brutalement compromis par un changement soudain.

Aucun de ces antagonismes n'est nouveau, mais ils sont tous devenus plus aigus. L'interdépendance fonctionnelle des institutions empêche de prédire les trajectoires de changement. La politique restera un facteur décisif. Aucun des deux pays n'a de parti libéral fort (Kitschelt 2000) ${ }^{11}$. Mais ils n'ont pas non plus de dirigeants capables de rajeunir le capitalisme non libéral sans nuire à l'égalité redistributive. Ceci est peut-être lié à la façon dont l'internationalisation change la nature de la puissance publique. « Des États encastrés dans les marchés, [...] ce n'est pas la même chose que des marchés encastrés dans les États » (Streeck 2000) : l'État n'a plus pour rôle de corriger le marché, mais plutôt celui d'y conformer l'économie. Ou cette incapacité reflète-t-elle plus simplement le manque de volonté politique face à l'attraction idéologique d'un modèle à l'américaine orienté par les actionnaires ? Celui-ci est favorisé par sa compétitivité renouvelée, par la part croissante de la population qui dépend pour ses retraites des revenus du capital et par les nouveaux modes de recrutement et de rémunération des directeurs (Dore 2000). Il apparaît ainsi peu probable ${ }^{12}$ que le capital politique nécessaire pour repenser le capitalisme non libéral et stopper son érosion par les marchés sera mobilisé13.

Traduit de l'anglais par Rachel Bouyssou

11. Le PLD au Japon et la CDU en Allemagne sont maintenus dans des positions modérées par plusieurs courants intérieurs, ainsi que par certains traits du système électoral, de la compétition politique et de l'organisation des ministères. 12. Il sera néanmoins intéressant d'observer des tentatives telles que celle de l'« Alliance pour l'emploi » tripartite allemande, visant à renégocier des réformes qui préservent les atouts du capitalisme non libéral tout en tentant de surmonter ses faiblesses les plus graves.

13. Cet article puise dans un projet de recherche (Streeck et Yamamura 1996) dont les résultats seront publiés dans Streeck et Yamamura 2000b. Pour plus de détails, voir www.mpi-fg-koeln.mpg.de/ gj 


\section{Bibliographie}

Aokı (Masahiko), 2000. Toward a Comparative Institutional Analysis. Cambridge (Mass.), The MIT Press, à paraître.

BOYER (Robert), 2000. " Germany and Japan : Two embedded capitalisms », dans STREeCK, YAMAMURA, 2000b.

DE JONG (Henk W.), 1996. " European capitalism between freedom and social justice », dans W. Bratton et al. (eds.), International Regulatory Competition and Coordination : Perspectives on Economic Regulation in Europe and the United States, Oxford, Clarendon Press.

DORE (Ronald), 2000. Stockmarket Capitalism, Welfare Capitalism : Japan and Germany versus the Anglo-Saxons, Oxford, Oxford University Press.

HASSEL (Anke), 2000. "The employment-welfare relationship in Britain and Germany ", dans B. Ebinghaus, Ph. Manow (eds.), Varieties of Capitalism, Londres, Routledge.

Hoshi (Takeo), KASHYAP (Anil), 1999. « The Japanese banking crisis: Where did it come from and how will it end ? ", NBER Working Paper $n^{\circ} 7250$,

http://www.nber.org/papers/w7250

JACKSON (Gregory), 2000. " Corporate governance in Germany and Japan : Liberalization pressures and responses ", dans StREECK, YAMAMURA, 2000b.

JACKSON (Gregory), VITOLS (Sigurt), 2000. "Pension regimes and financial systems : Between social security, market liquidity, and corporate governance ", dans B. EBBINGHAUS, Ph. MANow (eds.), Varieties of Welfare Capitalism, Londres, Routledge.

JIL (Japan Institute of Labor), 1995. "Rengo releases survey on employment check ", Japan Labor Bulletin 24 (7), juillet.

JÜRGENS (Ulrich), 2000. "Transformation and mutual interaction of the Japanese, American, and German production models in the 1990s ", dans Streeck, Yamamura, $2000 \mathrm{~b}$.

KITSCHELT (Herbert), 2000. "Competitive party democracy and political economic reform in Germany and Japan ", dans Streeck, Yamamura, 2000b.

KotTHOFF (Hermann), 1998. « Mitbestimmung in Zeiten interessenpolitischer Rückschritte. Betriebsräte zwischen Beteiligungsofferten und "gnadenlosem Kostensenkungsdiktat" ", Industrielle Beziehungen 5 (1).

LEHMBRUCH (Gerhard), 2000. "Discourses on socially embedded capitalism in Germany and Japan : Development and institutional framework ", dans STREECK, YAMAMURA, 2000a.

LÜTz (Susanne), 1999. « Vom koordinierten zum marktorientierten Kapitalismus ? Der deutsche Finanzsektor im Umbruch », dans R. CZADA, H. Wollmann (eds.), Von der Bonner zur Berliner Republik, Opladen, Westdeutscher Verlag.

MANOW (Philip), 2000. "Crisis and change in pension finance : Germany and Japan compared ", dans StREeCK, YAMAMURA, 2000b. 
MANOW (Philip), SEILS (Eric), 2000. « Adjusting badly : The German welfare state, structural change, and the open economy ", dans F.W. SCHARPF, V.A. SCHMIDT (eds.), From Vulnerability to Competitiveness. Welfare and Work in the Open Economy, Oxford, Oxford University Press, à paraître.

StREECK (Wolfgang), 2000. "Introduction : Explorations into the origins of non-liberal capitalism in Germany and Japan ", dans StreecK, YamamuRA, 2000a.

StREeCK (Wolfgang), 1997. « Beneficial constraints : On the economic limits of rational voluntarism ", dans R. BOYER, J.R. HOLLINGSWORTH (eds.), Contemporary Capitalism. The Embeddedness of Institutions, Cambridge, Cambridge University Press.

Streeck (Wolfgang), Yamamura (Kozo) (eds.), 2000a. The Origins of Non-Liberal Capitalism : Germany and Japan in Comparison, à paraître.

Streeck (Wolfgang), Yamamura (Kozo) (eds.), 2000b. Germany and Japan: The Future of Nationally Embedded Capitalism in a Global Economy, à paraître.

StReECK (Wolfgang), YamamURA (Kozo), 1996. Germany and Japan: The Future of Nationally Embedded Capitalism in a Global Economy (projet de recherche, non publié).

Thelen (Kathy), Kume (Ikuo), 2000a. " The rise of non-market training regimes : Germany and Japan compared", dans STREeCK, YAMAMURA, 2000a.

Thelen (Kathy), KUME (Ikuo), 2000b. « The future of nationally embedded capitalism : Industrial relations in Germany and Japan », dans StreecK, YAmAmURA, $2000 \mathrm{~b}$.

VITOLS (Sigurt), 2000. « From banks to markets : Liberalizing coalitions and financial reform in Japan and Germany », dans Streeck, Yamamura, 2000b.

YAmAMURA (Kozo), 2000. "Germany and Japan in a new phase of capitalism : Confronting the past and the future ", dans Streeck, Yamamura, $2000 \mathrm{~b}$. 